\title{
Proceso de producción de las cooperativas asociadas a PRODECOOP para la exportación de café orgánico a mercados preferenciales. 2014-2015
}

\author{
Zeyra Chavarría Espinoza² \\ Marycruz Ruiz Gutiérrez ${ }^{3}$ \\ Yessenia Lisseth Valdivia Gutiérrez ${ }^{4}$ \\ Beverly Castillo Herrera ${ }^{5}$
}

\section{RESUMEN}

El presente trabajo se enfoca en analizar el proceso de producción del café orgánico de los productores socios de la cooperativa PRODECOOP R, L. para el diseño de una estrategia que mejore los niveles de producción de café orgánico de calidad para la exportación. Según el nivel de conocimiento de esta investigación es aplicada y según el enfoque filosófico es cualitativa. La muestra la constituye el centro de cooperativas base PRODECOOP con sede en la ciudad de Estelí y su beneficio ubicado en el municipio de palacaguina. Las técnicas de recolección de datos fueron: investigación documental, observación y entrevistas. Las entrevistas se aplicaron a informantes claves de la empresa con experiencia en el proceso de producción a productores con varios años de conocimientos y exportación con la responsable. Los principales resultados obtuvimos que la relación de los productores asociados a PRODECOOP es pertinente ya que la mayoría son fundadores y laboran desde que inicio la empresa y les brinda una oportunidad de mantenerse y mejorar su calidad de vida. La certificación de las fincas es de gran importancia para la exportación porque le da seguridad al cliente del producto, ya que es un café orgánico y mediante el comercio justo se beneficia el productor. La mayoría de los productores cumplen con los parámetros de calidad que les exige PRODECOOP para obtener café en cantidad y calidad teniendo mejor rentabilidad en sus tierras. Los productores están satisfechos por formar parte de la cooperativa porque obtienen beneficios que apoya a mejorar su calidad de vida y esto también le favorece a PRODECOOP por que eleva su nivel de exportación y a la vez ayuda a la certificación de las fincas y así cumplir con los parámetros de calidad.

Palabras clave: Producción, exportación, mercado diferenciado.

Recibido: 10 de diciembre de 2015

Aceptado: 10 de junio de 2016

1 Este artículo se elabora a partir de la tesis titulada Proceso de producción de las cooperativas asociadas a PRODECOOP R,L para la exportación de café orgánico a merados preferenciales 2014-2015. Para optar al título de licenciada en administración de empresas en el año 2015 por la UNAN-MANAGUA, FAREM-ESTELI.

2 UNAN-MANAGUA, FAREM-ESTELI. Correo electrónico: zchavarria05@gmail.com

3 UNAN-MANAGUA, FAREM-ESTELI. Correo electrónico: maryolivares126@yahoo.com

4 UNAN-MANAGUA, FAREM-ESTELI. Correo electrónico: valdivia_yessenia@yahoo.es

5 Doctora en Ciencias Sociales por la Universidad del Zulia, Venezuela. Maestría en Ciencias Sociales por la Universidad de Guadalajara. Docente titular de la UNAN-Managua, FAREM-Estelí. Correo electrónico: beverly. castillo@yahoo.com 


\title{
Production process of cooperatives associated to PRODECOOP, for the exportation of organic coffee to preferential markets. 2014-2015
}

\begin{abstract}
The present work is aimed to analyze the process of production of organic coffee of producers associated to the cooperative PRODECOOP R, L., for the design of a strategy that improves the quality of production levels for the exportation of coffee. This is an applied, qualitative research. The sample consisted of the center of cooperatives PRODECOOP with headquarters in Estelí, and its branch located in Palacaguina. The data collection techniques were documentary research, observation and interviews. The interviews were applied to key informants of the company with wide experience in the production process. The main research results reveal that the relation of the producers associated to PRODECOOP is pertinent, since the majority of them are founders and work for it since the company was started. Moreover, it gives them the opportunity to improve their life quality. The majority of producers meet the quality criteria set by PRODECOOP to obtain coffee in quantity and quality, achieving a better profitability in their farms. The producers are satisfied for being part of the cooperative, as they obtain benefits that support their quality of life, as it increases the level of exportation, and quality criteria.
\end{abstract}

Keywords: Production, exportation, differentiated market. 


\section{INTRODUCCIÓN}

La Promotora de Desarrollo Corporativo de las Segovia PRODECOOP R, L nace en diciembre de 1993 como una Empresa Campesina de Servicios Múltiples, por iniciativa de los productores y profesionales comprometidos, con mejorar la calidad de vida de las familias de los pequeños productores (as) asociados a su cooperativa mediante su crecimiento empresarial $\mathrm{y}$ productivo y competir con calidad en el mercado internacional.

PRODECOOP desde el año 1993 se ha visto afectada por los bajo niveles de producción y la baja calidad del café orgánico, de igual manera la central en el ciclo productivo 2014-2015 se ve afectado por una baja en las exportaciones porque los productores no han cumplido con sus metas exigidas por la empresa, Lo que no genera los ingresos necesarios para sus socios y no están siendo suficientes para mejorar la calidad de vida. También están siendo limitados por la poca asistencia técnica porque PRODECOOP no cuenta con el personal necesario para brindar asesorías y capacitaciones. Otro problema es el cambio climático que ocasiona que el café no sea de calidad y también obstaculiza el transporte del café. La situación sociocultural que la mayoría de los socios tienen no ayuda a cumplir los parámetros de calidad que se les exige y por estas actitudes afectan las visitas de los técnicos, ya que los productores actúan de manera individualista. Otro aspecto es el poco financiamiento para la producción y mantenimiento de los cafetales que afecta de manera directa a los ingresos y la calidad de vida de los productores.

El reciente estudio contiene un análisis de las esenciales Fortalezas, Oportunidades, Debilidades y Amenazas que enfrenta PRODECOOP para proponer estrategias que generen oportunidades de desarrollo a la empresa, que servirán de guía para que los encargados tomen decisiones encaminadas a mejorar el proceso de producción para la exportación. Como objetivo más relevante se planteó analizar el proceso de producción del café de los productores socios de la cooperativa PRODECOOP R, L para el diseño de estrategia que mejoren los niveles de producción de café orgánico de calidad hacia mercados internacionales.

Esta investigación servirá de marco referencial a las cooperativas que trabajan con productores para que implementen los parámetros de certificación en sus fincas y conozcan la importancia de llevar a la práctica la asistencia técnica para cultivar un café de calidad para que se dé un incremento en la producción de café diferenciando su producto en el mercado. El desarrollo de esta investigación también proporciona información a los productores que todavía tienen problemas de cultura para que estén dispuestos a mejorar el desempeño sobre la producción y cumplan con la exigencia de PRODECOOP para el mercado y su exportación de café.

La base conceptual de esta investigación fueron cuatro ejes teóricos: Cooperativas, Proceso de producción del café orgánico, Proceso de certificación para la exportación, Mercado diferenciado. Para Fortanes (2002), cooperativa: es una asociación autónoma de personas que se han unido voluntariamente para formar una organización cuya administración y gestión debe llevarse según acuerden los socios. Según Severs(2005), el proceso de producción del café orgánico se da lo que es: producción del café, recolección, clasificación de la fruta, despulpado del fruto, desmusilaginado. Rafan (2007), la certificación es un proceso de sostenibilidad que incluye buenas prácticas agrícolas en las fincas, responsabilidad social empresarial, cumplimiento de leyes, normativas, mejoras continuas en la finca y oportunidad de mercado en el mundo del café. Herdez (2012), se refiere al mercado diferenciado que comprende la creación de diferencias en la oferta de productos, también se le conoce como segmentado que es donde la marca o empresa se dirige a varios segmentos de mercado. 


\section{MATERIALES Y MÉTODOS}

Según el uso de conocimientos esta investigación es aplicada porque estudia la problemática sobre el proceso de producción de las cooperativas asociadas a PRODECOOP R, L para la exportación de café orgánico de calidad a mercados preferenciales y se diseña una estrategia para mejorar dicho proceso. Es una investigación cualitativa porque se analiza a profundidad el proceso productivo de la cooperativa y se determina los diversos aspectos que intervienen en dicho proceso.

El universo de esta investigación lo constituye la central de cooperativas PRODECOOP R, L en la ciudad de Estelí y sus cooperativas asociadas en los diferentes departamentos (Nueva Segovia, Madriz y Estelí). El muestreo es no probabilístico porque no todo el universo tiene la misma probabilidad de ser seleccionado, se entrevistaron a: Informantes claves de la empresa, con amplios conocimientos en el proceso de producción, certificación y exportación (vice-gerente, jefe de producción y encargada de exportación); disposición de los informantes claves a ser entrevistados y participación de manera voluntaria en el estudio; y a productores de cooperativa asociada a PRODECOOP R, L con al menos un año de producir café orgánico.

Para cumplir con los objetivos planteados en la investigación se utilizaron cuatro técnicas de recolección de datos: entrevista, encuesta, guía de observación e investigación documental.

\section{RESULTADOS Y DISCUSIÓN}

\section{Relación de PRODECOOP R.L. con sus asociados}

La relación de esta cooperativa con sus asociados es pertinente, la mayoría son fundadores, están organizados desde el año 1993 lo que les brinda una oportunidad a esta empresa para mantener una afinidad con sus socios.

Uno de los aspectos importantes en esta empresa, es que gran parte de los socios pertenece a la directiva, tienen cargos y responsabilidades en cada una de sus cooperativas. Esto aporta en la toma de decisiones de la empresa ya que va en dependencia de la relación de estos, se obtienen de manera eficiente y eficaz, estos van de la mano para obtener mejores resultados y mantener su posicionamiento en el mercado.

Los productores asociados a la central están clasificados en tres categorías:

- Productores grandes: estos cuentan con los recursos monetarios suficientes, poseen tierras fértiles en cantidad abundante y por parte de PRODECOOP obtienen mayor confiabilidad a la hora de solicitar un crédito y son denotados como los mejores productores por entregar la mayor cantidad y mejor calidad de café.

- Productores medianos: estos poseen recursos monetarios limitados, poca tierra para trabajar, PRODECOOP brinda el financiamiento necesario y estos cumplen con la cantidad de café que deben entregar.

- Productores pequeños: estos no poseen los recursos monetarios suficientes, no cuentan con tierras propias y PRODECOOP no les da el financiamiento necesario por lo cual no cumplen con la entrega del producto.

Tabla $\mathrm{N}^{\circ} 1$. Tipos de productores asociados a PRODECOOP R.L.

\begin{tabular}{|c|c|c|}
\hline Tipos de productores & $\begin{array}{l}\text { Cantidad de } \\
\text { productores }\end{array}$ & $\%$ \\
\hline Productores Grandes. & 450 & $20 \%$ \\
\hline Productores Medianos & 720 & $25 \%$ \\
\hline Productores Pequeños & 1130 & $55 \%$ \\
\hline Total de Socios. & 2300 & $100 \%$ \\
\hline
\end{tabular}

Fuente: PRODECOOP R, L. 
Los socios productores se incorporan en la toma de decisiones de esta empresa de considera de manera efectiva, un ejemplo de esta es cuando la empresa quiere rotar al personal convoca a una junta a todos los socios para tomar decisiones.

Otro aspecto relevante es que son miembros de la directiva de su propia cooperativa o algún cargo o responsabilidad con PRODECOOP, es decir están dispuestos a trabajar por el bien común de los productores. Se relacionan de manera directa con la gerencia, los técnicos y otros socios de la asamblea general o en los grupos de trabajo que se organizan para el cumplimiento de la misión y visión de la empresa. Es importante destacar que al tomar una decisión se hace en conjunto los productores con la empresa para que no se dé un mal entendido y se escuchan ideas y opiniones.

Dentro de los beneficios que obtienen los socios al integrarse a PRODECOOP son:

- Crédito para la producción a corto, mediano y largo plazo.

- Servicios de asistencia técnica.

- Capacitación administrativa y productiva.

- Programas.

La central brinda el servicio de crédito o financiamiento a los productores para la producción y el mantenimiento del café, el monto se define de acuerdo a la necesidad del productor y su capacidad de pago. Los socios que solicitan crédito lo hacen en dos épocas; la primera para darle mantenimiento a la tierra entre junio y julio y la segunda en noviembre para la preparación del terreno húmedo y la plantación de la cosecha.

Los productores para optar a un crédito deben cumplir con los siguientes requisitos:

- Ser asociado a una cooperativa base de PRODECOOP.
- Estos créditos son solo para mantenimiento, renovación y apicultura.

- Tener entrega de café a PRODECOOP durante los últimos tres ciclos.

- No estar en estado moroso.

Las capacitaciones que se les brinda a los productores son orientadas por los técnicos a los representantes de cada cooperativa, quienes se encargan de trasmitírselos a los productores. Las capacitaciones son mensuales. Hay dos niveles de capacitación, una se hace con toda la cooperativa y la otra con cada representante de cooperativa para que luego les orienten a sus promotores e inspectores internos oficiales de control sobre la información abordada.

Los proyectos y programas que da la central son elaborados y gestionados por el Área de Desarrollo orientado por los técnicos quienes se encargan de impulsar los programas y proyectos.

Los productores asociados reciben beneficios sustanciales, que son valorados de gran provecho, utilizados para reinversiones, en sus actividades productivas. Esta organización se enfoca en la educación, formación académica en los hijos de los asociados.

Actualmente los principales programas que impulsa PRODECOOP son:

- Seguridad alimentaria en la Segovia.

- Rehabilitación de medios de vida a los asociados.

- Autogestión y seguridad alimentaria en cooperativas base.

- Política de equidad de género.

- Finca con fines de validación y trasferencia tecnológica.

- Renovación de cafetales.

- Programas de impacto social, en la administración y desarrollo. 
- Becas para secundaria y universidad.

- Paquetes escolares.

La certificación en los mercados preferenciales donde comercializa PRODECOOP R.L.

Las certificaciones son muy importantes para el proceso de certificación, desde que esta iniciativa se pudo en marcha se ha registrado un fuerte crecimiento, sobre todo en el mercado europeo. La certificación se centra en la promoción de buenas prácticas empresariales como componente importante para lograr la sostenibilidad. Su norma recoge criterios sociales y medio ambientales para la adopción de prácticas responsables en el cultivo del café y la gestión eficiente de las explotaciones. Los requisitos para la certificación que tiene PRODECOOP R, L. son:

- Numero de contrato con el cliente.

- Número del lote que lo proporciona PRODECOOP.

- Peso neto.

- Peso bruto.

- Lo que el cliente solicite.

Los parámetros de calidad que PRODECOOP debe cumplir al momento de exportar al mercado exterior son:

- La calidad del café que fue pactada, está es tratada con el cliente lo que va de acuerdo al tipo de preparación que le esté vendiendo si el café es con preparación europea solo puede llevar de uno a diez defectos en un lote de café y estos están pactados con el cliente.

- $\mathrm{Si}$ es una preparación americana puede llevar 20 defectos como grano mordido, quebrado y caracoles.

- Si el cliente pide un café de tasa excelencia esa calidad de café se le venderá ya que no tiene que llevar ningún defecto.

- La calidad la establece el comprador y el vendedor, esto depende de la preferencia del cliente.
Esta empresa empezó en el mercado internacional para mantener sus clientes fijos, con seguimiento del personal de exportación, ha desarrollado una serie de habilidades que le ha permitido posicionarse en el mercado diferenciado con la venta del café. Se tiene responsabilidad, compromiso, buena comunicación, participación en ferias, visitas a clientes, como formas de darse a conocer en el mercado. Esta organización tiene buena comunicación y confianza con sus clientes.

Para exportar a mercados preferenciales se debe estar registrado en el CETREX (Centro de Trámites de las Exportaciones), cuyos requisitos que son: Presentar documento de personería jurídica de la empresa, representante legal, poder de representante legal, firma de las personas que van a firmar documento ante el CETREX. Cuando se exporta por primera vez, primero se hace un contrato con el cliente y se registra en el CETREX.

Al realizar el embarque se coordina con la naviera, se elabora y se recibe del cliente la instrucción de embarque donde se indica el puerto, naviera, consignatario, note fax, destino, si va inspeccionado o no y otros detalles incluidos en la factura.

Se tiene que tramitar un certificado fitosanitario, ante un laboratorio certificado en Nicaragua, este documento se llama certificado de calidad (esto es indispensable). Cuando se tienen todos los documentos y trámites realizados se puede realizar el embarque. La factura se elabora de acuerdo a la fijación de precios que se haya dispuesto con el cliente, en algunos casos el precio pactado se define en el mes que se va embarcar y después se fija el precio de acuerdo a la bolsa de Nueva York.

El café se carga en el beneficio, se envía al agente aduanero quien hace todos los tramites, cuando el contenedor llega a Corinto, Las Manos o Peñas Blancas, Cuando el barco zarpa del puerto la naviera entrega el documento original, por ejemplo: se le hace 
un borrador legal BL (bil o freyri) donde dice quién es el embarcador y consignatario. La naviera avisa al contacto por teléfono o correo que el café está por llegar para que prepare la documentación, declare ante aduana y estén preparados. El cliente tiene un lapso de dos días para retirar el contenedor, si no lo retira incurrirá en multa.

Los volúmenes de exportación se miden por sacos de café que son cien libras de café por cada quintal esa es la unidad de medida para hacer el embarque. Los contratos son de 250 hasta 310 sacos por contenedor, pero hay contratos de solo 100 sacos y hay micro lotes que pueden ir en un contenedor desde 20 a 10 sacos hasta formar un volumen grande. Hay que tomar en cuenta que los costos son altos y por más volumen de café es más económico.

En el 2015 los niveles de exportación de PRODECOOP se mantienen, se ha cumplido con la demanda de los clientes. Se han exportado entre 480 a 600 contenedores, el nivel de exportación no ha bajado porque el café orgánico es de mayor rentabilidad para la PRODECOOP. (Ver tabla N²)

Tabla $\mathrm{N}^{\circ}$ 2. Los países adonde exporta PRODECOOP R.L.

\begin{tabular}{lrrr}
\hline \multicolumn{1}{c}{ Paises } & $\begin{array}{r}\text { Cantidad de } \\
\text { café a exportar }\end{array}$ & $\begin{array}{c}\text { Precio a } \\
\text { exportar }\end{array}$ & $\begin{array}{c}\text { Total } \\
\text { Dinero }\end{array}$ \\
\hline Europa & $27,540 \mathrm{qq}$ & 200 euros & $5,508,000$ \\
Bélgica & $5,200 \mathrm{qq}$ & 200 euros & $1,040,000$ \\
Inglaterra & $5,508 \mathrm{qq}$ & 200 euros & $1,101,600$ \\
Dinamarca & $5,600 \mathrm{qq}$ & 200 euros & $1,120,000$ \\
Irlanda & $5,100 \mathrm{qq}$ & 200 euros & $1,020,000$ \\
Suecia & $6,132 \mathrm{qq}$ & 200 euros & $1,226,400$ \\
Total Países & & & $\mathbf{1 1 , 0 1 6 , 0 0 0}$ \\
Europeos & $\mathbf{5 5 , 0 8 0 q q}$ & & \\
$\begin{array}{l}\text { Países de } \\
\text { América del } \\
\text { norte }\end{array}$ & $64,260 \mathrm{qq}$ & 250 dólares & $16,065,000$ \\
$\begin{array}{l}\text { EE.UU ( } \\
\text { Houston, } \\
\text { Oakland, }\end{array}$ & & & \\
New york) & $51,408 \mathrm{qq}$ & 250 dólares & $12,852,000$ \\
& & &
\end{tabular}

Canadá 12,852qq 250 dólares $3,213,000$

Total EE.UU $128,520 q q$ $32,130,000$

PRODECOOP tiene clientes fijos y con relación de largo plazo. Tienen su red de distribución a donde se exporta y se trabaja con importadores, que cuentan con su red de tostadores a nivel nacional, E.E.U.U. y Europa, poseen sus propias cafeterías y lo venden a los supermercados en tiendas de conveniencia; tienen su segmento de mercado al que le venden el café.

\section{Tabla N`3. Utilidad que obtiene PRODECOOP} R, L al exportar café orgánico

\begin{tabular}{ll}
\hline Total de compra de café & $\mathrm{U} \$ 23,868,000.00$ \\
Total de incentivos & $\mathrm{U} \$ 4,590,000.00$ \\
Total de gasto por exportar & $\mathrm{U} \$ 1,924,000.00$ \\
Total & $\mathrm{U} \$ 30,382,000.00$ \\
Total de café a exportar & $\mathrm{U} \$ 43,146,000.00$ \\
\hline Valor del café a exportar menos & $12,764,000$ \\
Gastos Totales = utilidad & \\
\hline
\end{tabular}

Fuente: PRODECOOP R.L., 2015

\section{Cumplimiento de los parámetros de certificación de productores asociados a PRODECOOP R, L.}

Los requisitos que deben cumplir los productores para aplicar a una certificación: que sea dueño de la tierra, la certificación se hace individual y si el productor tiene más de 200 manzanas paga su certificación. Los pequeños productores que poseen de 2 a 5 manzanas pagan la certificación en grupo y se le llama "grupo comunitario" porque son de escasos recursos económicos.

Los productores trabajan con tres tipos de certificaciones que son:

- Certificación orgánica: exigen requisitos como el cuido al medio ambiente y no uso de químicos.

- Certificación de mercado justo: PRODECOOP pertenece a un grupo llamado "Comunitario 
La Segovia" donde se reúnen todos los socios orgánicos. La certificación se hace con los diferentes grupos comunitarios que tienen ingresos mayores a 5,000 córdobas.

- Sello de pequeños y medianos productores: en el caso de sello de pequeños productores que se puede aplicar agroquímicos de baja toxina. Para comprobar que los productores están cumpliendo se hacen inspecciones anuales.

Los productores están clasificados según los números de manzana de las que son propietario y de igual manera por la cantidad de quintales de café producido en grandes, medianos y pequeños.

La central garantiza la calidad y hace cumplir los parámetros utilizandolaasistencia técnica. Secuentacon doce técnicos agrónomos que atienden semanalmente a cada una de las cooperativas, se encargan de la supervisión de las fincas de los productores y garantiza que los productores utilicen adecuadamente los recursos orgánicos, en los diferentes tipos de café. En el caso del café convencional, se explica el proceso de preparación, se revisa si hay algún tipo de enfermedad y si se encuentra alguna deficiencia en la planta se orienta como combatirla y mejorar las fallas del cultivo.

Los productores que reciben asistencia técnica, de los competentes asignados. Los califican como eficaces en sus tareas asignadas, y les permite elevar la calidad y cantidad de la producción de café.

Implementar los parámetros de calidad total garantiza producir un café con buenas características organolépticas como aroma, sabor, acides; todas las características debidas del café. Los productores deben cumplir con dos modalidades o parámetros de calidad para que su café sea aceptado los parámetros son: que el café orgánico sea de calidad, con un 10\% de humedad y mohoso, el grano este entero, no quebrado, el color no sea negro, no esté sucio que no contenga terrones o broza, no lleve granos verdes.

\section{Incidencia de los beneficios en el cumplimiento de la calidad de la producción.}

Los beneficios, húmedos y secos garantizan que el café este limpio de impurezas y vender un producto de calidad que satisfaga al consumidor. El café de primera que se exporta tiene un proceso donde se selecciona y se clasifica. Así mismo puede salir un café de segunda que no es completamente orgánico porque le falta un subproceso de selección.

Para seleccionar el tipo de café, se estiba en polines y para diversificación del tipo de café se rezaga de esta manera: el café orgánico en sacos verdes, el café de convención en color blanco, el café en transición y el T3 convencional en saco color naranja o rosado, el café en transición a orgánico T1Y T2 en color amarillo.

Beneficio húmedo: El beneficio húmedo representa un eslabón importante en la calidad. Inicia con el despulpado del café maduro, se despulpa con máquina y comienza su fermentación en pilas de cemento o madera, separación de granos, limpieza de impurezas y lavado, se orea, se escoge y se lleva al centro de acopio. La calidad depende de un buen corte solo de granos maduros y darle un buen punto al fermentado para evitar granos manchados y pelados. Este proceso se lleva como promedio 2 días.

Beneficio seco: El subproceso número uno es la recepción del café que proviene del beneficio húmedo, verifica la cantidad, el peso y las condiciones físicas que viene el producto. El café en el beneficio seco se ubica en un patio segmentado del beneficio. Un patio de cementó para el café orgánico y patios naturales para el café no orgánico se coloca un plástico en la parte de abajo.

Este café va lotificado cuando llegan los sacos de café los envían al patio con una documentación que permite luego aplicar trazabilidad se verifica si el café viene con algunos síntomas de enfermedades, si es así se les 
realiza monitoreo constante a través del laboratorio de control de calidad quienes extraen muestras y se realiza el análisis de rendimiento para la captación de café según el tipo de problema en el caso que continua con daños se identifica y se le clasifica una calidad inferior. Una norma es el color del café así se puede distinguir el producto que está en observación.

\section{Proceso de trillado y clasificado}

Una vez aprobado un lote de café, se traslada en pergamino al área de máquina. El trillado lo realiza una máquina llamada trilladora, que es un cilindro que por fricción le quita partículas de pergamino y el café queda al desnudo. Luego el café llega a la catadora de succión y su función es sacar el grano muy liviano y granos quebrados. El café sigue su ruta, a las máquinas de movimiento vibratorio que clasifica el café en tres calidades: primera, segunda y tercera. Dependiendo de la preparación, el café pasa a la máquina clasificadora de tamaño, que tiene varias bandejas agujeradas que clasifican siete tamaños o calidades de café.

Para dar la calidad final, el café se traslada a unas máquinas clasificadoras por color, esta elimina los granos claros y granos muy oscuros. El café de segunda se regresa por las maquinas antes mencionadas la que no se utiliza, es el trillado cuando el café ha realizado todo el recorrido se extrae una muestra que se envía al laboratorio, para verificar el control de calidad, con la muestra enviada antes y con el café del nuevo proceso.

Luego de este proceso el café pasa al supervisor de calidad quien determina si la calidad es acertada o si amerita una nueva limpieza, cuando los granos se consideran defectuosos y se debe mejorar.

El último proceso es la mezcla, empaque y despacho. Se fusiona el café de segunda y tercera calidad en el mezclador o pulverizador. Y se empaca en bolsa de una libra para su comercialización local que el café de palo y cafecito Segoviano.
El producto terminado espera el contenedor. Hay otra área llamada logística de exportación que hacen el vínculo con la naviera la que envía el camión, este se verifica, es parte del proceso de despacho y se comprueba que el contenedor no tenga orificios o agujero y se procede a enviar.

\section{Proceso de producción del café}

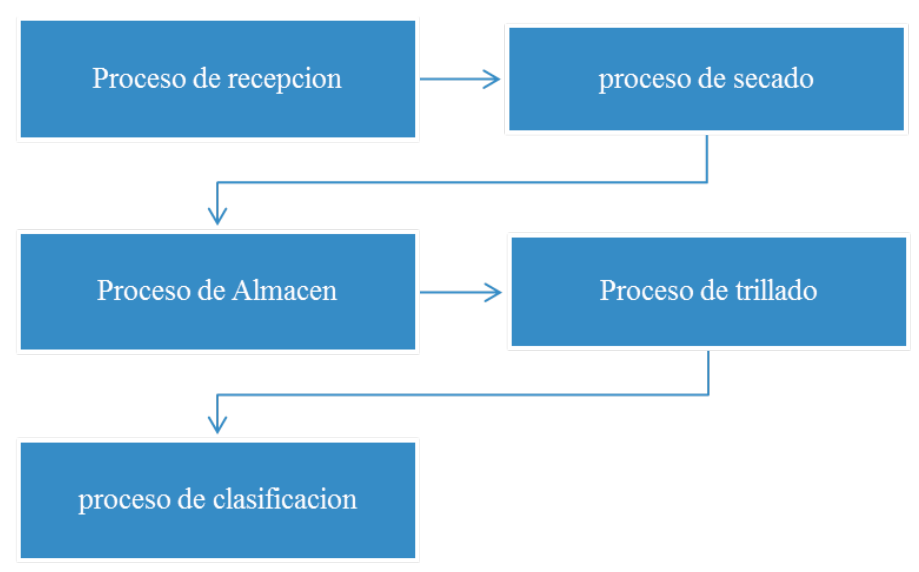

Causas que dificultan el cumplimiento de los parámetros de calidad entre los productores.

- Los gastos del proceso de certificación en visitas de inspector interno a la parcela, se llevan un control de campo de todas las actividades y llenan un registro donde comprueban si cumplió con lo orientado.

- Es difícil el Cumplimiento de los estándares de calidad exigidas por la certificadora, porque no se lleva seguimiento por parte de los productores por lo tanto no producen en un $100 \%$ café de calidad.

- Para que la producción de café sea de calidad se debe tener un buen manejo de la planta sin uso de químicos y para la recolección se debe hacer un buen corte. 
Estrategias para mejorar los niveles productivos para la exportación.

\section{Matriz FODA de PRODECOOP R.L.}

A partir de los resultados de la presente investigación se analiza las principales Fortalezas, Oportunidades, Debilidades y Amenazas de la central de cooperativa

\begin{tabular}{lll}
\hline \multicolumn{1}{c}{ Fortalezas } & Oportunidades \\
\hline - $\begin{array}{l}\text { Experiencia laboral } \\
\text { productiva. }\end{array}$ & $\begin{array}{l}\text { Aumento de la } \\
\text { demanda de café }\end{array}$ \\
- Cuentan con equipos & orgánico. \\
de tecnología & PRODECOOP \\
avanzada. & conserva el \\
Poseen manual de & posicionamiento en \\
certificación de las & el mercado exterior. \\
fincas. & Alianza con \\
Seguimiento de & organización \\
programas sociales & de cooperativas \\
para los productores. & externas. \\
Estricto control de & \\
calidad para los & \\
productores. & \\
\hline
\end{tabular}

\section{Debilidades}

- Limitados fondos para el financiamiento.

- Poco personal para la asistencia técnica.

- Desmotivación de los productores en el cumplimiento de los parámetros.

\section{Amenazas}

- Cambio climático.

- Preferencia de los pequeños productores hacia otros mercados.

- Incorporación de nuevos ofertantes de café certificado al mercado diferenciado.

- Bajos niveles de producción de los productores.

- Situación sociocultural de los productores.

Estrategias para mejorar la calidad de la producción.

Línea $\mathrm{N}^{\circ} 1$ : Motivación a los productores para mejorar la calidad del café desde su producción. Objetivo general: Fomentar a los productores de la producción de calidad.

Línea $\mathbf{N}^{\circ}$ 2: Creación de una línea de crédito diseñada para pequeños productores para el mantenimiento de sus cafetales. Objetivo general: Garantizar financiamiento a los pequeños productores.

Línea $\mathbf{N}^{\circ}$ 3: Elevar los niveles de producción de café tomando en cuenta la calidad y cantidad para el aumento de la demanda. Objetivo general: Establecer un plan de seguimiento y control de actividades del proceso de producción de café orgánico para llevar control de calidad con los productores.

Línea $\mathbf{N}^{\circ}$ 4: Desarrollo y crecimiento organizativo. Objetivo general: Fortalecer organizativamente cada una de las cooperativas.

\section{CONCLUSIONES}

La central de cooperativas PRODECCOP R, L. ha logrado posicionarse en el mercado diferenciado y se mantiene en constante desarrollo, sin embargo es necesaria la participación de los productores en la producción de café de calidad para la exportación.

Para que el café ingrese al mercado diferenciado, es necesario que PRODECOOP cuente con una certificación que lo avale, para aplicar a ella el productor debe cumplir con los parámetros de calidad de producción exigido por la certificadora. El equipo técnico de PRODECOOP se encarga de capacitar a los socios de cada cooperativa y la aprobación de la certificación es realizada por la certificadora OIC.

La calidad del café orgánico, depende de la variedad del café que se siembra como el catimorra, el terreno 
debe ser adecuado, el grado de humedad tiene que estar estable, mantener un almacenamiento adecuado y realizar buenas prácticas durante la recolección de fruto, despulpe, fermentación, beneficio húmedo y del beneficio seco.

PRODECOOP exporta entre 480 a 600 contenedores de café anual a EEUU y Europa considerando que se han mantenido el nivel de exportación. Representa un $80 \%$ al comercio justo y el $20 \%$ al mercado comercial.

\section{BIBLIOGRAFÍA}

Fortanes, D. (2002). Importancia de las cooperativas. Obtenido de: www.comerciohispano.com

Severs, R. (2008) Manual de buenas prácticas de manufactura. Obtenido de: www.mag.go.cr/ bibliotecavirtual/a00205.pdf

Herdez, O. (2012). Marketing Diferenciado. Obtenido de: http://www.procafe.com.sv/menu/ ArchivosPDF/HojaTecnicaOrganicoYComercio. Pág., 35 